\title{
Scale-up and coverage of Avahan: a large-scale HIV- prevention programme among female sex workers and men who have sex with men in four Indian states
}

- Supplementary table published online only at http:// sti.bmj.com/content/vol86/ issue 1

${ }^{1}$ Family Health International, New Delhi, India ${ }^{2}$ Karnataka Health Promotion Trust, Bangalore, India ${ }^{3}$ Pathfinder International, Maharashtra, India ${ }^{4}$ Hindustan Latex Family Planning Promotion Trust, Hyderabad, India ${ }^{5}$ ndia HIV/AIDS Alliance, Hyderabad, India ${ }^{6}$ Tamil Nadu AIDS Initiative-Voluntary Health Services, Chennai, India ${ }^{7}$ Department of Community Health Sciences, University of Manitoba, Winnipeg, Canada ${ }^{8}$ CARE, Delhi, India

\section{Correspondence to}

Mrs Ruchi Verma, 16, Sunder Nagar, New Delhi 110003, India; rverma@fhiindia.org

Accepted 14 November 2009

\author{
Ruchi Verma, ${ }^{1}$ Abhijeet Shekhar, ${ }^{1}$ Sharmistha Khobragade, ${ }^{1}$ Rajatashuvra Adhikary, ${ }^{1}$ \\ Bitra George, ${ }^{1}$ Banadalkoppa M Ramesh, ${ }^{2}$ Virupax Ranebennur, ${ }^{1}$ Sudipta Mondal, ${ }^{3}$ \\ Rajesh Kumar Patra, ${ }^{4}$ Sandhya Srinivasan, ${ }^{5}$ A Vijayaraman, ${ }^{6}$ Sushena Reza Paul, \\ Nabesh Bohidar ${ }^{8}$
}

\begin{abstract}
Objective Documenting the implementation of a public health programme as per its design is critical to interpretation of results from survey-led outcome and impact evaluation activities, the authors describe the scale-up and coverage of large-scale HIV-prevention services provided to female sex workers (FSWs) and high-risk men who have sex with men (HR-MSM) during the first 5 years of the Avahan programme in India.

Methods Implementing NGO partner-generated denominator estimates from 70 districts were used to estimate the programme's intended coverage. Routine programme-monitoring data until December 2008 were used to describe the service and commodity availability, service utilisation to generate internal estimates of coverage. Coverage was validated in few districts using data from a cross-sectional survey.
\end{abstract}

Results In December 2008, the estimated denominators for intended services were about 217000 FSWs and 80000 HR-MSM. By January 2007, 79\% of eventual total clinics and $75 \%$ drop-in centres were established, and $83 \%$ of eventual peer educators were active. By month 48, sufficient condoms to cover all estimated FSW commercial sex acts were distributed free. By month 60, $75 \%$ of the estimated denominator intended to be covered was met monthly. $86 \%$ of FSWs and $67 \%$ of HRMSM ever contacted had used sexually transmitted infections services at least once. Cross-sectional survey generated coverage results suggest that programmemonitoring data provide a proxy to coverage of services. Conclusion Avahan's monitoring data show that Avahan achieved infrastructure scale by year 3 and high contact coverage through peers and with commodities by year 5 of implementation as per the design.

\section{INTRODUCTION}

Experts believe that the global HIV epidemic can be contained by scaling up comprehensive HIVprevention programmes complemented by high levels of coverage and quality, and of sufficient duration (for most at-risk populations relevant to local transmission dynamics). ${ }^{1}$ With an estimated 2.31 million people living with HIV, India has a concentrated HIV epidemic. ${ }^{2-4}$ High-risk groups (HRGs) in India include female sex workers (FSWs), men having sex with men (MSM) and injectingdrug users (IDU) as well as bridge group populations such as male clients of sex workers. ${ }^{3} 4$ In late 2003, the Bill and Melinda Gates Foundation funded the Avahan India AIDS Initiative, an HIV-prevention programme for HRGs in the four southern states of Andhra Pradesh, Tamil Nadu, Karnataka and Maharashtra, and two north-eastern states of Manipur and Nagaland estimated to account for over $80 \%$ of country's estimated HIV infections in 2002. ${ }^{4-6}$ The core Avahan package of HIV-prevention services included behaviour-change communication delivered through peer educators and outreach staff, programme-supported free clinical services to treat sexually transmitted infections (STI), commodity provision (free condom distribution, condom social marketing and needle exchange) and referral linkages to other services. ${ }^{5-7}$ In addition, stigma, violence and barriers to accessing entitlements were addressed through structural and community mobilisation interventions. ${ }^{8} 9$ In December 2007, Avahan was providing HIVprevention services to over about 300000 FSWs, high risk MSMs and IDUs through seven state-level lead implementing partners in 82 districts in the six states. ${ }^{5}$

One of Avahan's goals is to impact the Indian HIV epidemic by achieving high coverage (over $80 \%$ ) with effective HIV-prevention services to HRGs and bridge groups across the intervention areas. 510 The current paper aims to epitomise and document the Avahan programme scale-up and coverage of FSW and HR-MSM populations in the four southern states. ${ }^{11}{ }^{12}$ Coverage is examined using a framework which considers the availability, accessibility and acceptability of appropriate quality services that result in high levels of utilisation by the target population. ${ }^{13} 14$ This paper examines the availability, utilisation and coverage of HIV-prevention services for HRGs. Accessibility and acceptability indicators were not captured through routine monitoring data. However, accessibility was achieved by placing HIV-prevention services in proximity to mapped solicitation points in consultation with FSWs and HR-MSM. Acceptability of services was maximised by involving FSWs and HR-MSM as part of the outreach and facilities (drop-in centre (DIC) and STI clinics) management teams. ${ }^{579}$ Companion papers in this supplement address the question of quality of Avahan's STI clinical services and condom social marketing. 1516 


\section{Programme description}

Between December 2003 and March 2004, grants were awarded to six lead implementing partners in the four southern states (22 districts in Andhra Pradesh, 17 districts in Karnataka, 13 districts each in Maharashtra and Tamil Nadu). The lead implementing partners in turn awarded subgrants to local NGO (implementing partners)..$^{5}$ In August 2005, a final grant was awarded to support interventions in four additional districts in Karnataka and Maharashtra. In late 2007, a few districts were reallocated in consultation with the government of India bringing 70 districts under Avahan Programme in 2008. In December 2003 and March 2004 , separate grants were awarded for programmes with male clients, for condom social marketing and franchised STI clinical services, and for interventions with long distance truckers. Districts were chosen to complement existing government or other donor-supported targeted intervention programming so as to collectively achieve high coverage of the HRG population in the four states. In December 2008 Avahan was the sole implementer of HIV-prevention services in 43 districts (solo districts) and provided services in parts of the remaining districts along with government of India or other donors (major or minor districts). ${ }^{17}$

The major activities at the district level included (1) recruitment and training of outreach and clinic staff; (2) validation of estimated numbers of HRGs and mapping of solicitation points; (3) setting up a supply-chain system for free condoms in consultation with State AIDS Control Societies; (4) setting up monitoring and reporting systems; and (5) determining locations and establishing DICs and programme-owned STIs clinics in consultation with HRGs. ${ }^{5}$ The initial focus of the programme in the four southern states was mainly FSWs except for two lead implementing partners (Tamil Nadu and Maharashtra) who included HR-MSM from the beginning. By mid-2005, all other lead implementing partners working in the states had initiated interventions for HR-MSM.

\section{METHODS}

Six broad areas of enquiry formed the basis of the analysis in this paper: (1) denominator of the populations intended to be reached; (2) need for commodities provision (condoms); (3) availability of HIV-prevention services and condoms over time; (4) utilisation of services over time by HRGs; (5) internal estimates of service coverage over time using denominator and programme-generated utilisation data; and (6) external validation or 'evaluated coverage' of HRGs by programme services through survey data at specific time points.

As many NGOs and service sites served both FSWs and HRMSM, availability of service sites over time is not presented separately, whereas utilisation and coverage data are presented separately for the two HRGs. The sections below describe in more detail the data sources and analytical approaches.

\section{Data sources and assumptions}

Data sources include: (1) size estimate data to establish the denominators for HRGs; (2) programme-monitoring data and (3) a first round of cross-sectional survey among core and bridge populations through the Integrated Behavioural and Biologic Assessment (IBBA) conducted over 2006-2007 in a subset of districts which provides external validation of population level coverage. $^{18}$

\section{Denominators and HRG intended coverage}

Intended coverage was defined as the number of HRG individuals estimated to be in the geographic area where the local implementing NGO had been contracted to establish services by Avahan, government of India or other donors. The HRG denominator estimation process is described in table 1 , and statelevel data are listed in Web table 1. For the purpose of this analysis, the denominator determined in December 2008 is assumed to be the intended denominator of coverage for the entire time period examined. This is reasonable, as there have been no major natural disasters, economic dislocations or civil strife that might be associated with sudden changed numbers of FSW except for the closing of the dance bars in Mumbai. ${ }^{19} 20$ However, evidence suggests that efforts by police did not lead to a decrease in the number of sex workers but only leads to shifts in the solicitation venues. ${ }^{21}$

\section{Programme-monitoring data}

The Avahan programme monitoring information system (MIS) evolved over the first 3 years of programme implementation. ${ }^{17}$ At the start of the project, each lead implementing partner defined their indicators and established data-collection mechanisms. Between late 2004 and 2005, with the support of an Avahan-wide monitoring and evaluation grantee (Family Health International) a standard set of core indicators was established, and by late 2005, reports were generated on the core set of indicators by the NGOs. Between July and August 2006, the core set of indicators were expanded. The analysis here uses information from January 2005 and assumes all values to be zero in January 2004; linear interpolation was used to impute the midyear values for the indicators for 2004. Integrity of data was ensured by the lead implementing partners.

\section{IBBA}

The IBBA was designed to measure changes in key behavioural and biological indicators among HRGs. ${ }^{18}$ The first round of the IBBA covered 29 of the 83 districts where Avahan is being implemented and covered 25162 respondents, including FSW in 25 districts, HR-MSM in 11 districts, IDU in five districts, male clients of sex workers in 12 districts, and long-distance truck drivers on four national highway segments or route categories. ${ }^{18}$ In this analysis, IBBA data on exposure to outreach services, STIclinic utilisation, and mean and median number of partners for commercial sex by typology of FSW have been used.

\section{Analytical approach}

\section{Availability and utilisation of services}

Availability of services was examined using time trend analysis of hard infrastructure (ie, proportion of total NGOs contracted, proportion of total STI clinics and DICs established) and soft infrastructure (ie, number and ratio to target population of active trained peer educators and outreach workers) (table 1). Utilisation of services was examined by analysing the number of unique individuals and percentage of HRG population contacted though outreach (monthly and ever contacted), accessing STI clinical services (monthly and ever visited) and distribution of commodities (condoms) provided by the programme (table 1).

\section{Condom need}

Adequacy of condom distribution was assessed using data on estimated condom need among HRGs. The overall estimated condom need for FSWs was calculated using the number of FSWs by typology (based on solicitation point) as recorded in the programme-monitoring data and the number of reported commercial sex partners by typology of FSWs in the IBBA (table 1). This was not calculated for HR-MSM, as the necessary information was not available. 
Table 1 Indicators with definitions used in analysis

\begin{tabular}{ll}
\hline Area & Data source/indicator(s) \\
\hline Denominator & Programme data: number of FSWs and HR-MSM in
\end{tabular}
geographic area

Availability

\section{MIS}

1. NGOs subcontracted

2. DICs established

3. Static project owned clinics established

4. Number of active peer educators (FSW and HR-MSM) and outreach staff, and the ratio of peer educator to the community member

5. Number of condoms distributed to FSW and HR-MSM both for free and through lead implementing partner condom social marketing by NGOs

Utilisation

MIS

1. Number of individuals ever contacted

2. Number of individual contacted monthly

3. Number of individual ever visiting the clinic for STI services

4. Number of individual visiting the clinic for STI services monthly

Estimated condom need

MIS - number of contacts by typology of FSWs based on solicitation venue served during outreach IBBA - mean number of reported commercial sex partners both regular and occasional for FSWs and mean number of male partners for HR-MSM

Validity of MIS data

MIS - (1) contacted this month, (2) ever visited the clinic IBBA-(1) contacted by peer in the year, (2) visited a project STI clinic in last year (except Karnataka; see comments)
Comments/description

Avahan HRG size estimates: at the start of an intervention in a district or subdistrict, NGOs conducted a formal external mapping and size estimation exercise. Some state-level lead implementing partners updated these numbers on a regular basis, every 12 to 18 months, using programme data; some did formal size estimation exercises; and some used programme data alone to estimate numbers through subtracting individuals currently in the programme (determined on a 3 to 6 month basis depending on the lead implementing partner) from individuals ever registered. ${ }^{17}$ Size estimates are separate for FSWs and HRMSM. The size estimate used in this analysis is the number as of December 2008 District HRG size estimates: size estimates are available from the Project Implementation Plans for third National AIDS Control Programme. Data are based on previous mapping exercises conducted by the states and where applicable Avahan size data.

1. Some NGOs provided services uniquely to FSWs or HR-MSM, but the majority provided services to both. NGOs are not stratified by population served.

2. DICs are places for HRG members to gather, meet and rest in comfortable, safe environment. DICs were established with community input regarding location, hours and available services, and managed by community.

3. In addition to providing static STI clinics (eg, clinics in a permanent location) which were chosen, staffed and operated with input from the community, other STI services delivery sites were provided. These sites included fixedtime, fixed-location mobile clinics, preferred private providers and health camps. These other STI service sites are not included in the infrastructure analysis because reporting was not standardised across lead implementing partners but are included in the STI utilisation indicator.

4. Outreach contacts are made by peer educators and the outreach staff. The number of peer educators is available as FSWs and HR-MSM separately but the number of outreach staff is present as a total figure. The target ratio per programme guidelines of a peer to community members is 1:50.

5. Lead implementing partners distributed condoms free to the FSWs and HRMSM through peer educators, outreach staff, clinics, depots and social marketing. In 2006, state-level lead implementing partners were instructed to ensure that enough condoms were distributed to meet the estimated condom needs of the individual FSWs and HR-MSM at the implementing site level. The method to estimate this need was determined at the state-level lead implementing partner level.

(1) and (3) All individuals at a site were given an identification number used at clinical services and for outreach. Ever contacted or came to the clinic services is determined by this number locally and reported in aggregate.

(2) and (4) Individuals contacted during outreach or seen in clinic are aggregated at the site level on a monthly basis and reported. Number of unique individuals contacted is known only on a monthly basis. Number of times an individual contacted over time or the frequency of contacts cannot be determined from central MIS. Programme targets for monthly outreach contacts varied across partners but were at a minimum at least one contact per month. Programme targets for routine STI clinic consultations were once a quarter, resulting in a target of $33 \%$ per month attending the clinic.

For FSWs, IBBA reports commercial partner number by three FSW solicitation typologies - brothel-based, home-based, public-based. For the analysis of condom requirement, all sex work typologies recorded in the MIS that were not brothel-based or home-based were classified as public-based (street, tamasha, lodge, bar based, private and others).

For FSWs, total sex acts are calculated on a state level using MIS reported FSW typology, and mean and median partner number by typology by state reported in the IBBA. State level numbers are summed for overall commercial sex acts by FSW. At the time of calculation, one commercial partner $=$ one sex act $=$ one condom is assumed for FSWs. Estimation of total condoms needed for FSWs was 34 condoms per FSW per month to cover all commercial sex acts using the median reported number of sexual partners according to partner type.

For HR-MSM - the commodity need was not calculated, as the relevant required information was not available.

1. All districts except Karnataka reported receiving services from a peer educator in the last month (IBBA) compared with percentage contacted through outreach in the last month (MIS) (number over 2008 district denominator). In Karnataka districts (Belgaum, Bellary, Shimoga), the reference period for the IBBA question was the last 1 year.

2. For all districts except Karnataka, reported visiting programme STI clinics in the past 1 year (IBBA) compared with the percentage that ever visited programme clinics (number over 2008 district denominator). For Karnataka districts, the IBBA question was last 6 months. 


\section{Validity of programme monitoring coverage data}

For reported exposure from the IBBA, the comparison is limited to IBBA districts where Avahan was the sole implementer in the district and where there were no interventions prior to the Avahan programme. Exposure to outreach services and STI clinics reported during the IBBA survey period were compared with programme-monitoring data from the same time period (last month survey in the district) in the districts analysed (table 1).

\section{RESULTS}

Overall, of the total estimated denominator for HRG in the four southern states, the Avahan programme's intended coverage was estimated to be $66 \%$ for FSW and $70 \%$ for HR-MSM. It varied between 55 and 79\% for FSWs and between 50 and $87 \%$ for HRMSM within the four southern states (Web table 1). Under Avahan, 116 local NGOs were contracted to implement programmes with HRGs, and 520 DICs and 302 programme-run STI clinics were established by December 2008 in the four southern states. About $85 \%$ of the total NGOs were contracted, and about $80 \%$ of static STI clinics and $75 \%$ of DICs were established by January 2007 (figure 1A).

The number of active (ie, currently working) peer educators and outreach workers rose from 385 to 5810 and 296 to 1272 respectively between January 2004 and December 2008 (figure 1B). By January 2007, the programme had above $85 \%$ of the eventual PEs (ie, peer educators working as of December
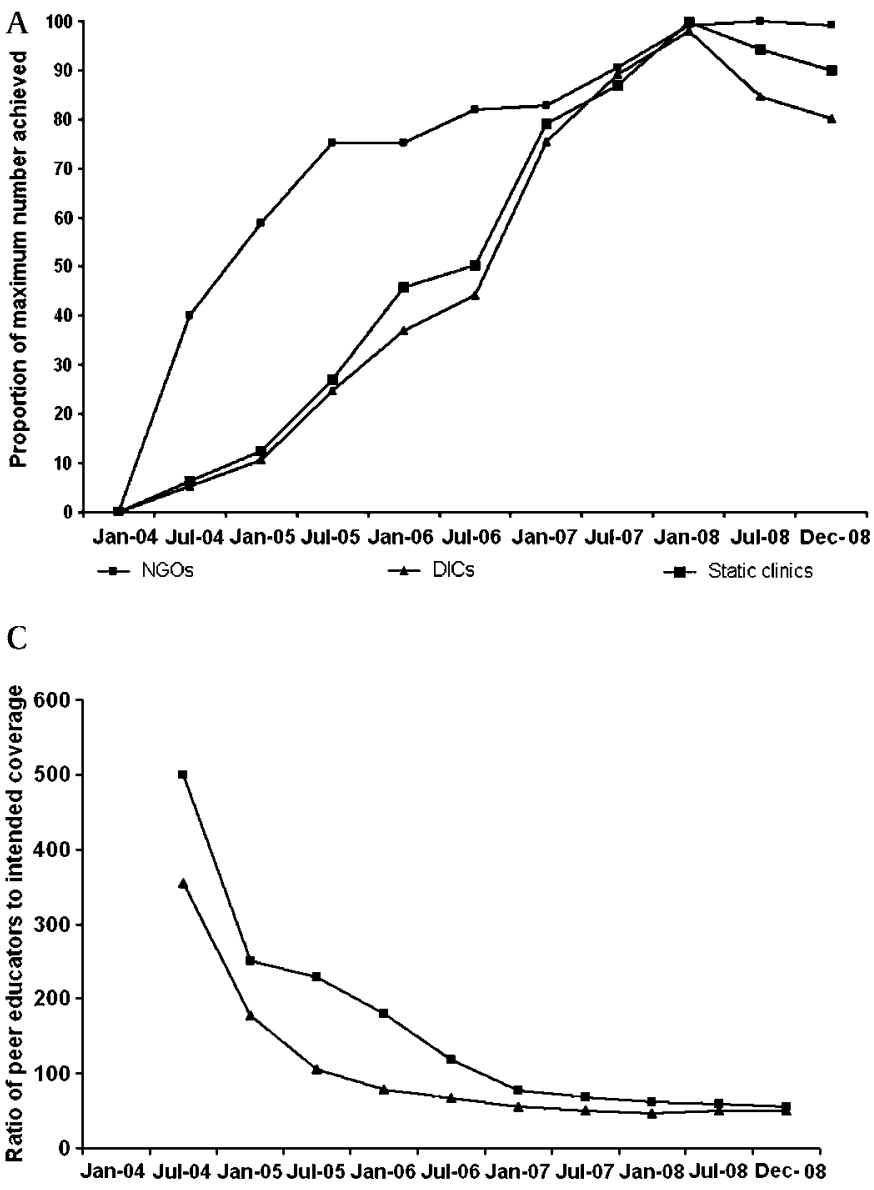

$\star$ Ratio of PE to intended coverage (FSW) - Ratio of PE to intended coverage (HR-MSM)
2008). In January 2007, the ratio of peer educator to HRG population was 1:55 for FSW and 1:76 for HR-MSM and decreased steadily to 1:50 for FSWs and 1:55 for HR-MSM in December 2008 (figure 1C).

Condom distribution (free and social marketed) by the statelevel lead implementing partners rose steadily after January 2006. The estimated requirement for 34 condoms per month per FSW for commercial partners was met through direct distribution by January 2008 (figure 1D). In December 2008, condom distribution by state-level lead implementing partners was 41 condoms per month per FSW and 34 condoms per month per HR-MSM.

The number of individuals ever contacted through outreach and ever visited the clinic for STI services is shown in figure 2A. By December 2008, about 350000 FSWs and 100000 HR-MSM had been contacted through outreach activities. The proportion of the total denominator of HRG intended to be covered that were ever contacted through outreach and ever visited STI services was $160 \%$ and $139 \%$ respectively for FSWs and $122 \%$ and $82 \%$, respectively, for HR-MSM (figure $2 \mathrm{~B}$ ). The proportion of the total denominator intended to be covered that were contacted monthly through outreach reached approximately $50 \%$ in January 2007 and was over 75\% for both FSWs and HRMSM by December 2008 (figure 2C). Out of those ever contacted by December 2008, 86\% FSWs and 67\% HR-MSM had visited the programme STI clinics at least once. The proportion of HRG members of the total denominator of intended coverage visiting

B

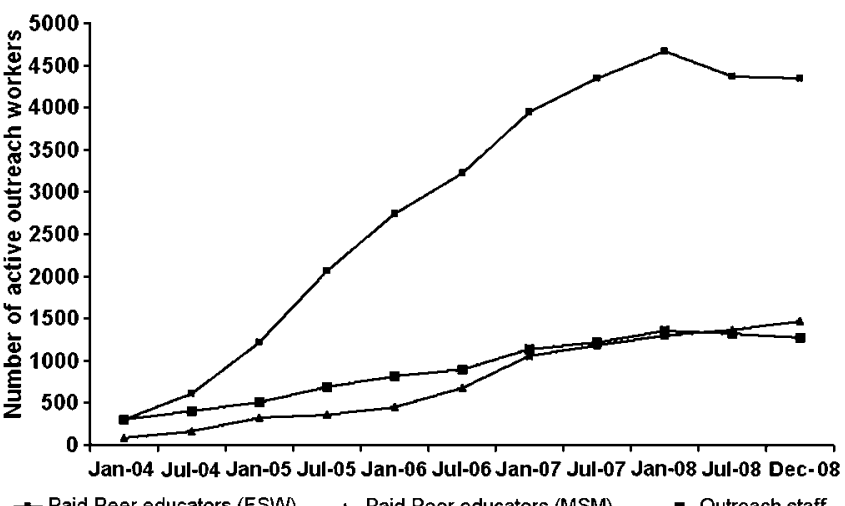

$\rightarrow$ Paid Peer educators (FSW) $\rightarrow$ Paid Peer educators (MSM) $\rightarrow$-Outreach staff

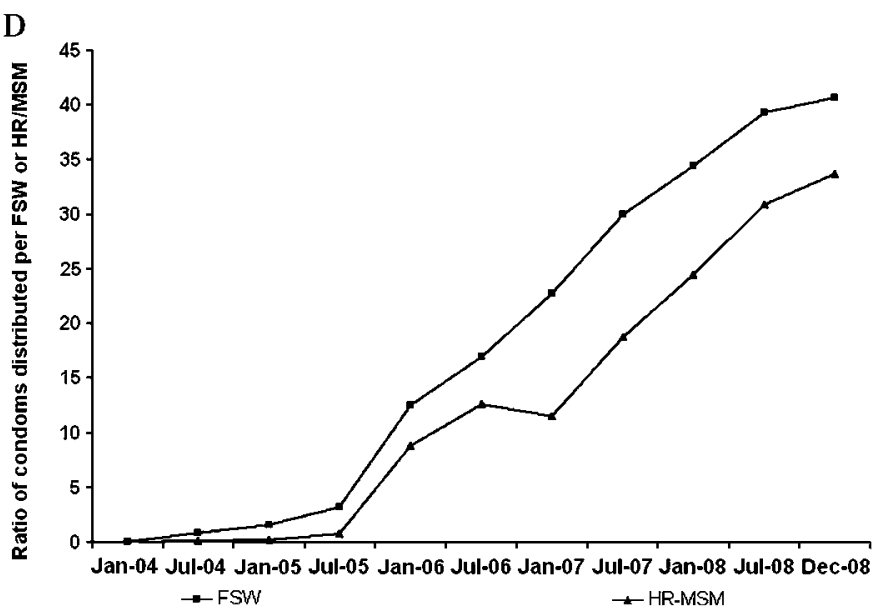

Figure 1 (A) Percentage of maximum number of NGOs contracted drop-in centres (DICs) and static STI clinical services established over time. (B) Total number of active outreach workers, female sex worker (FSW) peer educators and high-risk men who have sex with men peer educators. (C) Ratio of active peer educators to total target denominator intended to be covered for FSWs and high-risk men who have sex with men (HR-MSM). (D) Ratio of estimated condom distribution (free and socially marketed) per FSW and HR-MSM per month by state-level lead implementing partners using total target denominator intended to be covered throughout. 
STI programme clinics each month was about $25 \%$ for FSWs and over $15 \%$ for HR-MSM (figure 2D) by December 2008, compared with the programme target of $33 \%$ of HRG visits per month (one STI consultation or routine check-up per HRG every 3 months).

Table 2 compares reported exposure data from IBBA for FSW and HR-MSM for solo Avahan districts against equivalent coverage as internally estimated for the same time period by programme-monitoring data. Survey measurement of coverage for FSWs and HR-MSM populations via peer outreach and visits to programme STI clinics is at least as high as coverage estimated using programme-monitoring data and denominators.

\section{DISCUSSION}

Based on this analysis of the programme-monitoring MIS, Avahan was implemented as designed and achieved hard (clinics, DIC) and soft (trained outreach staff including peer educators) infrastructure scale in 3 years after the programme start and was meeting three-quarters of the estimated denominator of intended coverage every month in 5 years after programme start. The reasons for the time lag were due to the time required to process grants to local NGOs; completion of local situation assessments to design projects locally; and to build rapport with the community. ${ }^{72}$

Direct condom distribution to FSWs by the state-level lead implementing partners through free distribution or social marketing grew to cover the estimated needs for all commercial sex acts for FSWs estimated in the intended coverage in 4 years after programme start. The increase in the absolute number of condoms distributed was proportional to the increase in FSWs contacted by the outreach staff. This analysis reflects only those condoms provided directly to the FSWs from the NGOs but does not capture condoms available through the Avahan supported targeted condom social marketing programme, other socially marketed condoms or retail outlets in India. ${ }^{23}$ While condom sales are poor indicators of the prevalence of condom use, the wide condom availability in India suggests that condoms directly distributed to FSWs represent only part of the available condoms for commercial sex. ${ }^{24} 25$ The number of condoms distributed is based on the peer and NGO records, and dumping of condoms to achieve targets cannot be ruled out.

The Avahan programme resulted in strong outreach activities conducted at the field level as evidenced by the fact that a total of $70 \%$ of HRGs were contacted every month by outreach staff by the fourth year of the programme. The strength of outreach is probably due to the high ratios of the number of peer educators to project staff, which increased the ability of HIVprevention interventions to identify, engage and follow-up HRGs, and motivate them to attend STI clinics and achieve the intended coverage. This analysis did not examine peer educator

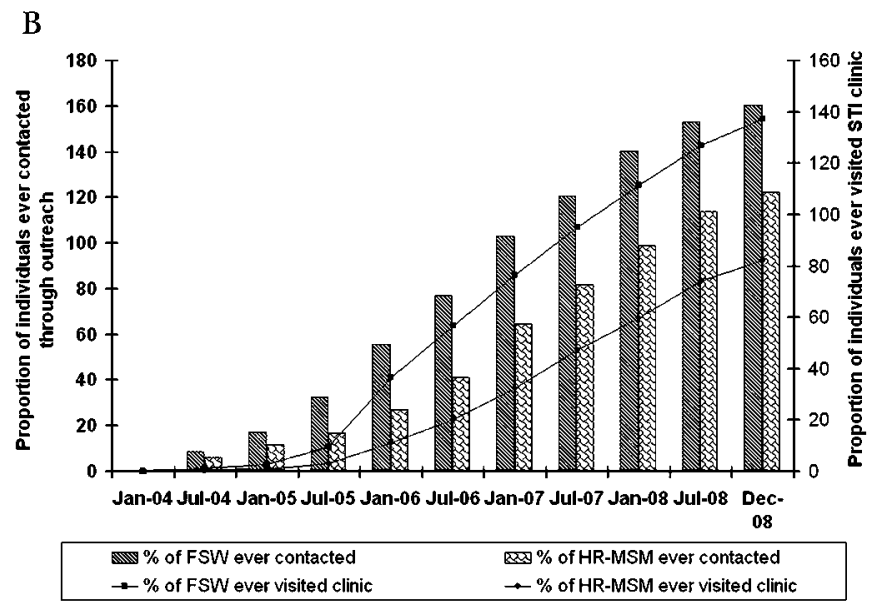

D

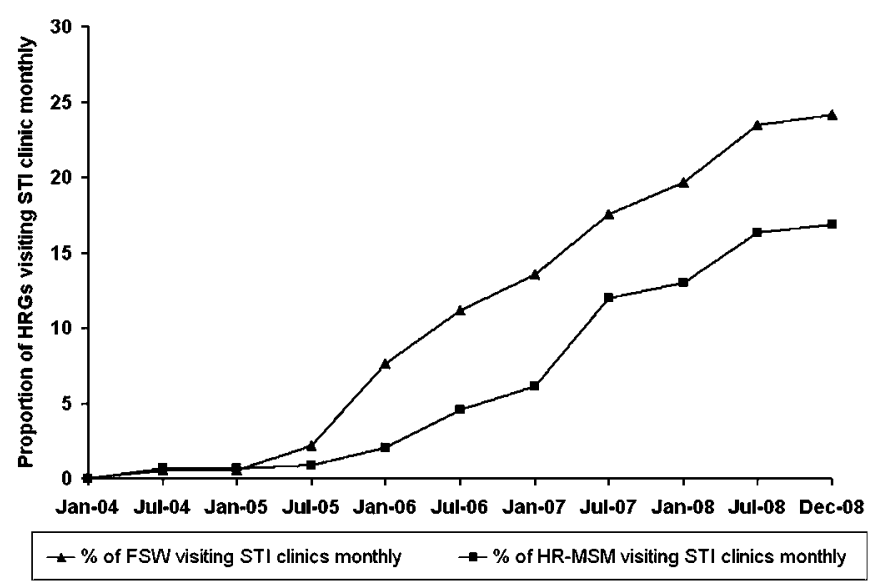

Figure 2 (A) Number of high-risk group members (HRGs) ever contacted through outreach and ever visited the clinic for STI services by female sex workers (FSW) and high-risk men who have sex with men (HR-MSM). (B) Percentage of HRGs based on 2008 denominator intended to be covered ever contacted through outreach and ever visited the clinic for STI services by female sex workers (FSW) and HR-MSM. (C) Percentage of total number of HRGs (based on 2008 denominator intended to be covered) contacted monthly through outreach services. (D) Percentage of total number of HRGs (based on 2008 denominator intended to be covered) visiting the clinic for STI services monthly. 
Table 2 Comparison of monitoring information system (MIS) and Integrated Behavioural and Biologic Assessment (IBBA) coverage data in districts where Avahan was the first and sole implementer

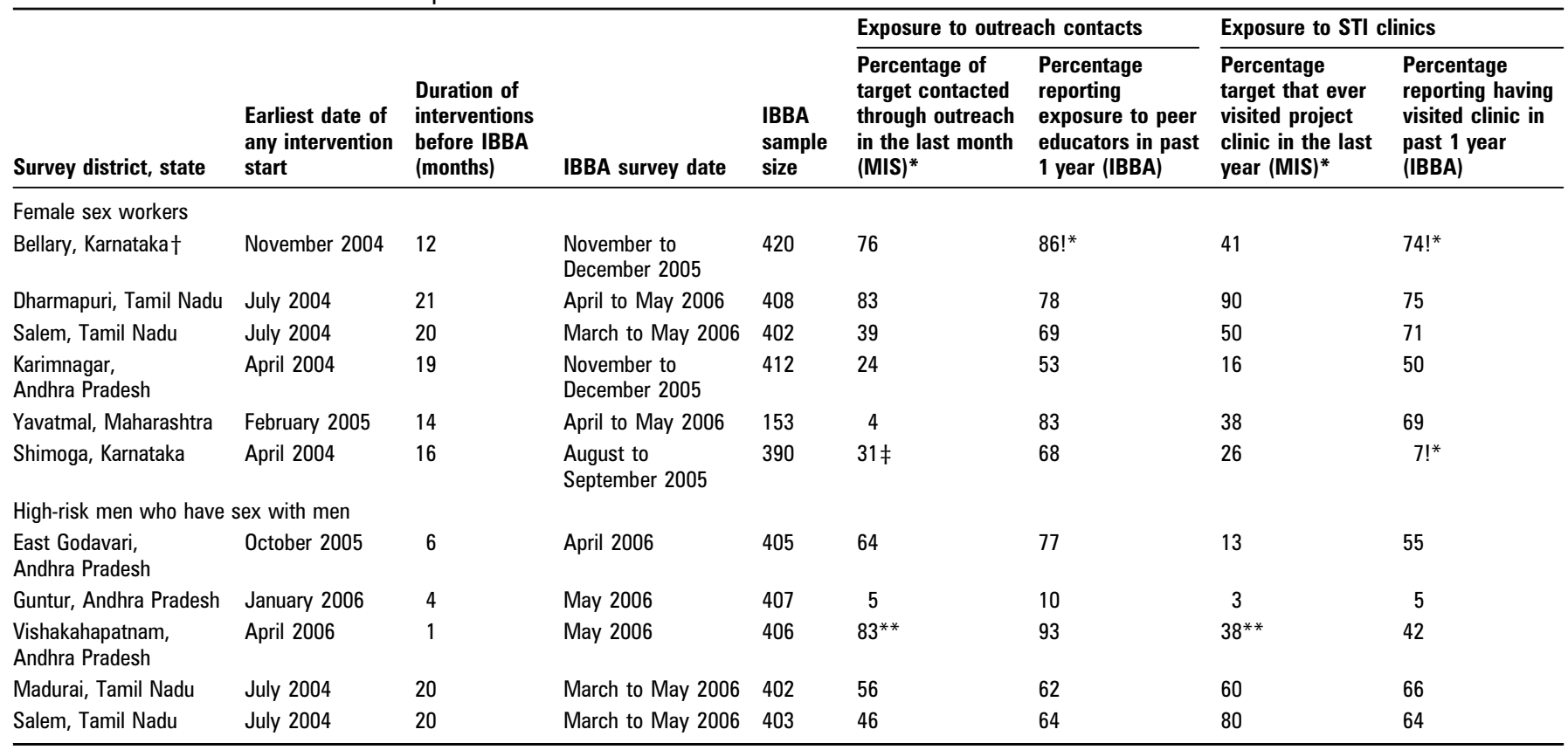

*December 2008 district denominator used for calculation.

!*IBBA questionnaire for Karnataka is slightly different from the other states. The period is 6 months instead of a year.

$\ddagger$ The first available data are from October 2006, indicated here.

**The first available data are from December 2006 indicated here.

turnover or the work load of peer educators which could affect the quality of outreach services and the ability to achieve intended targets in a timely manner.

The IBBA presented an opportunity to validate the coverage data estimated from the programme-monitoring data in the MIS. The IBBA data collected in late 2005 and early 2006 showed a higher proportion of FSWs and HR-MSM who reported contact by outreach staff than the MIS data with the sole exception of one district. The reason for the exception could be that the IBBA was conducted when the monitoring system was not yet stable. In some districts, exposure measured by programme-monitoring data was vastly lower than the IBBA exposure data, suggesting that the programme monitoring may have been weak in these districts, social desirability bias in the IBBA responses, or lack of clarity in the questions asked in the IBBA survey. It also may reflect the highly mobile nature of the FSWs in these districts and states, and presence of longstanding non-Avahan HIV-prevention interventions in some districts in these states. The mobility, and entrance and exit from the sex trade are consistent with the MIS data from which we estimate that $160 \%$ of the FSW population intended to be covered has been contacted by an outreach worker at least once. Over $100 \%$

\section{Key messages}

- Demonstrates the scale-up and coverage of Avahan Program among female sex workers and men who have sex with men in four southern Indian states.

- Provides information on the size of infrastructure as well as program indicators like condom distribution and STI consultations over time.

- Presents the trend on the outreach and clinical services provided to the High risk groups. results for the ever contacted FSW through outreach and STI services appears to account for the high mobility of FSW. This also accounts for some of the difference given the relatively close estimate of coverage figures in the MIS and IBBA for HR-MSM where interventions prior to Avahan were almost non-existent. ${ }^{4}$ The programme-monitoring data do not appear to overstate coverage and are a reasonable proxy for coverage based on this MIS analysis, as the programme was not fully scaled up during the first round of IBBA data collection. ${ }^{10}$

A key limitation of this analysis is its reliance on size estimates that cannot be validated externally. Size estimation of HRGs (through direct or indirect methods) is a difficult exercise due to the mobile, hidden and hard-to-reach nature of these population groups. ${ }^{26} 27$ Size estimates represent a number at one point in time and do not capture the likely variations in local numbers that occur over the programme period. Recent data from India suggest that there is a high degree of mobility; about $70 \%$ of FSWs in Andhra Pradesh report having moved across districts in the previous 2 years. ${ }^{28}$

The Avahan programme MIS and IBBA presented a unique opportunity to understand scale-up and coverage achievements for a large-scale HIV-prevention programme using routine monitoring and survey measures. Monitoring data are necessary for programme management and decision-making, and thereby contribute to good implementation. ${ }^{23}$ In measuring outcome and impact, good-quality monitoring trend data are critical to document that the programme was implemented as per its design without which evaluating programme effectiveness will not be meaningful. ${ }^{11}$ Scale-up of programme infrastructure and programme activities varied across states. Assessments of coverage presented here show that the programme was able to achieve high, consistent levels of coverage within 5 years of initiation of intervention. While the current analysis can be further enhanced by more in-depth analysis at district and regional level, there is sufficient evidence of scale-up and 
population coverage by the programme to ensure subsequent outcome and evaluation results will be plausible.

Acknowledgements We would like to thank A Tangri, P Chandrasekaran and G Dallabetta, from the India office of the Bill \& Melinda Gates foundation for providing details on Avahan implementation start-up and for their review and comments on the manuscript. We would like to thank B Mahapatra, P Goswami and L Ramakrishnan from the India office of Family Health International for providing for their review and comments on the manuscript. We would also like to thank $S$ Noriega from Family Health International, Asia Pacific Regional Office, Bangkok, Thailand, and MA Weaver from Family Health International, Headquarters, North Carolina, USA for reviewing and providing comments on the manuscript.

Funding This activity was funded by The Bill \& Melinda Gates Foundation. The views expressed herein are those of the authors and do not necessarily reflect the official policy or position of The Bill \& Melinda Gates Foundation.

Competing interests None.

Provenance and peer review Not commissioned; externally peer reviewed.

\section{REFERENCES}

1. Bertozzi S, Laga M, Bautista-Arredondo S, et al. Making HIV prevention programs work. Lancet 2008;372:831-44.

2. Pandey A, Reddy DC, Ghys PD, et al. Improved estimates of India's HIV burden in 2006. Indian J Med Res 2009;129:50-8.

3. Moses S, Blanchard J, Kang H, et al. AIDS in South Asia: understanding and responding to a heterogeneous epidemic. Washington, DC: World Bank, 2006. http:// siteresources.worldbank.org/SOUTHASIAEXT/Resources/Publications/448813 1155152122224/southasia aids.pdf.

4. Chandrasekaran P, Dallabetta G, Loo V, et al. Containing HIV/AIDS in India: the unfinished agenda. Lancet Infect Dis 2006;6:508-21.

5. Bill and Melinda Gates Foundation. New Delhi Avahan-the India AIDS initiative: the business of HIV prevention at scale. New Delhi, India. 2008. http://www. gatesfoundation.org/avahan/Documents/Avahan HIVPrevention.pdf.

6. Steen R, Mogasale V, Wi T, et al. Pursuing scale and quality in STI interventions with sex workers: initial results from Avahan India AIDS Initiative. Sex Trans Infect 2006;82:381-5

7. Blanchard JF, Bhattacharjee $P$, Kumaran $S$, et al. Concepts and strategies for scaling up focused prevention for sex workers in India. Sex Trans Infect 2008;84: ii19-23.

8. Bill and Melinda Gates Foundation New Delhi. Managing HIV prevention from the ground up: Avahan's experience in peer led outreach at scale in india, New Delhi, India. 2008 http://www.gatesfoundation.org/avahan/Documents/ Avahan PeerOutreach.pdf.

9. Reza-Paul S, Beattie T, Syed HR, et al. Declines in risk behaviour and STI prevalence following a community-led HIV preventive intervention among female sex workers in Mysore, India. AIDS 2008;22(Suppl 5):S91-100.

10. Chandrasekaran P, Dallabetta G, Loo V, et al. Evaluation design for large-scale HIV prevention programmes; the case of Avahan, the India AIDS initiative. AIDS 2008;22 (suppl 5):S1-15.
11. Habicht JP, Victora CG, Vaughan JP. Evaluation designs for adequacy, plausibility and probability of public health programme performance and impact. Int J Epidemio 1999;28:10-18.

12. Rychetnik L, Frommer $\mathrm{M}$, Hawe $\mathrm{P}$, et al. Criteria for evaluating evidence of public health interventions. J Epidemiol Community Health 2005;56:119-27.

13. Technical consultation on effective coverage in health systems. In: Murray CJL, Evans DB, eds. Health systems performance assessment: debates, methods and empiricism. Geneva, Switzerland: World Health Organization, 2003;8:125-33. http:// whqlibdoc.who.int/publications/2003/9241562455.pdf.

14. Bryce J, Victora CG, Habicht JP, et al. The multi-country evaluation of the integrated management of childhood illness strategy: lessons for the evaluation of public health interventions. Am J Public Health 2004;94:406-15.

15. Mogasale V, Wi TC, Das A, et al. Quality assurance and quality improvement using supportive supervision in a large scale STI intervention with sex workers, men who have sex with men/transgenders and injecting-drug users in India. Sex Transm Infect 2010;86(Suppl 1):i83-8.

16. Piot B, Mukherjee A, Navin D, et al. Lot quality assurance sampling for monitoring coverage and quality of a targeted condom social marketing programme in traditional and non-traditional outlets in India. Sex Transm Infect 2010;86(Suppl 1): i56-61.

17. Bill \& Melinda Gates Foundation. New Delhi, India. Use it or lose it: how Avahan used data to shape its HIV prevention efforts in India. 2008. http://www. gatesfoundation.org/avahan/Documents/Avahan UseltOrLooselt.pdf.

18. Saidel T, Adhikary R, Mainkar M, et al. Baseline integrated behavioral and biologica assessment among most at-risk populations in six high-prevalence states of India: design and implementation challenges. AIDS 2008;22(Suppl 5):S17-34.

19. Ummul Saba. Of entertainment, dance bars and ban. http://www.dayafterindia.com/ july106/bar.html (accessed 30 December 2009).

20. Press Trust of India. Dance bars banned in Maharashtra. Web site. Available at: http://www.expressindia.com/news (accessed 30 December 2009).

21. Harcourt C, Donovan B. The many faces of sex work. Sex Transm Inf 2005;81:201-6.

22. King EM, Behrman JR. Timing and duration of exposure in evaluations of social programs. World Bank Res Obs 2009:24:55-82.

23. Lipovsek V, Mukherjee A, Navin D, et al. Increases in self-reported consistent condom use among male clients of female sex workers following exposure to an integrated behaviour change programme in four states in Southern India. Sex Transm Infect 2010;86(Suppl 1):i25-32.

24. Bradley J, Moses S, Blanchard JF, et al. Assessing reported condom use among female sex workers in southern India through examination of condom availability. Sex Transm Infect 2010;86(Suppl 1):i44-8.

25. Meekers D, Van Rossem R. Explaining inconsistencies between data on condom use and condom sales. BMC Health Services Research 2005;5:5.

26. Family Health International [FHI] Arlington, Virginia, institute for HIV/AIDS, UNAIDS/ 03.36E, USAID Cooperative Agreement No HRN-A-00-97-00017-00, Estimating the size of populations at risk for HIV: issues and methods. 2003,03.36E. 2003. http:// data.unaids.org/Publications/External-Documents/EstimatingPopSizes en.pdf.

27. Selvaraj V, Gupte MD, Adhikary R, et al. Appropriateness and execution challenges of three formal size estimation methods for high-risk populations in India. AIDS 2008;22 (Suppl 5):S137-48.

28. Saggurti N, Verma RK, Reddy H, et al. Patterns of migration/mobility and HIV risk among female sex workers: Andhra Pradesh 2007-08. New Delhi: Population Council, 2008 http://www.popcouncil.org/pdfs/India FSWHIVAndhra.pdf. 